\title{
The structural panorama of L-asparaginases includes an alien from nitrogen-fixing bacteria
}

\author{
M. Jaskolski ${ }^{1,2}$, J. Loch $^{3}$, M. Gilski ${ }^{1,2}$, B. Imiolczyk ${ }^{2}$ \\ ${ }^{1}$ Department of Crystallography, Faculty of Chemistry, A. Mickiewicz University, Poznan, Poland, \\ ${ }^{2}$ Center for Biocrystallographic Research, Institute of Bioorganic Chemistry, Polish Academy of Sciences, Poznan, Poland, \\ ${ }^{3}$ Faculty of Chemistry, Jagiellonian University, Cracow, Poland \\ mariuszj@amu.edu.pl
}

L-Asparaginases from bacterial periplasm (e.g. EcAII) have high L-asparagine affinity and are used as potent antileukemic drugs. Plants possess a different, Ntn class of asparaginases, which are also found in bacteria (e.g. EcAIII). It was predicted $\sim 20$ years ago that Rhizobium etli, a bacterial symbiont of legume plants that is capable of nitrogen fixation, will possess yet another, R.etli-type Lasparaginase. The crystal structure of this enzyme, ReAII, reveals a dimeric protein that is indeed completely different from the EcAII and EcAIII prototypes, with structural resemblance to some serine $\beta$-lactamases and glutaminases. The presumed active site is organized around S48, which is surrounded by three tightly H-bonded water molecules and is further H-bonded to N134. Near-by there is a tandem of Cys residues coordinating a zinc cation. The coordination sphere is completed by a water molecule and a Lys side chain. Another Lys residue penetrates the active site to provide an H-bond link to S48. C225 of this Cys-rich protein also bears an unknown posttranslational modification.

Keywords: amidohydrolases; leukemia; Rhizobium etli; posttranslational modification

Work supported by NCN grant 2020/37/B/NZ1/03250. 